\title{
Essais
}

Revue interdisciplinaire d'Humanités

Hors-série 3 | 2016

Usages critiques de Montaigne

\section{Approches rhétoriques des Essais}

Déborah Knop

\section{(2) OpenEdition}

Journals

Édition électronique

URL : http://journals.openedition.org/essais/6686

DOI : 10.4000/essais. 6686

ISSN : 2276-0970

\section{Éditeur}

École doctorale Montaigne Humanités

\section{Édition imprimée}

Date de publication : 1 novembre 2016

Pagination : $30-43$

ISBN : 979-10-97024-00-0

ISSN : 2417-4211

Référence électronique

Déborah Knop, «Approches rhétoriques des Essais », Essais [En ligne], Hors-série 3 | 2016, mis en ligne le 26 février 2021, consulté le 17 mars 2021. URL : http://journals.openedition.org/essais/6686 ; DOI : https://doi.org/10.4000/essais.6686 


\section{Approches rhétoriques des Essais}

\section{Déborah Knop}

Étant donné le déclin de l'enseignement de la rhétorique au cours du $\mathrm{XIX}^{\mathrm{e}}$ siècle, et sa quasi-disparition au moment du grand essor des recherches critiques en France (début $\mathrm{XX}^{\mathrm{e}}$ siècle), pendant longtemps, la rhétorique n'a pas été mise à contribution des lectures de Montaigne ${ }^{1}$. C'est la recherche des quatre dernières décennies, sous l'impulsion de deux colloques sur Montaigne et la rhétorique en particulier $^{2}$, qui a permis de nouveau de la mobiliser pour lire les Essais.

Le rejet de la rhétorique est un leitmotiv des Essais qui a fait couler beaucoup d'encre ; rappelons que l'art rhétorique est enveloppé dans un rejet de l'art en général, au même titre que la médecine, par exemple ${ }^{3}$. Montaigne réduit à plusieurs reprises la science rhétorique à des figures médiocres, qui la dévalorisent : Guillaume Poyet par opposition au Cardinal Du Bellay (I, 10, 39) $)^{4}$, les ambassadeurs de Samos, à Cléomène (I, 26, 170), Lysias, à Socrate lui-même (III, 12, 1052-1054)5. Or, ce que Montaigne désavoue, voire fustige, par ces binômes antithétiques, ce n'est pas exactement l'art de l'éloquence, mais la "rhétorique

1 Voir par exemple Essais de Michel de Montaigne, avec les notes de tous les commentateurs, éd. J.-V. Le Clerc, Paris, Lefèvre, 1826. Bien que J.-V. Leclerc ait traduit et publié l'ensemble des discours de Cicéron, les notes de cette édition des Essais ne sont pas de teneur rhétorique.

2 Rhétorique de Montaigne, Actes du colloque de la Société des Amis de Montaigne (14-15 décembre 1984), éd. Frank Lestringant, Bulletin de la Société des Amis de Montaigne, juil.-déc. 1985, VII ${ }^{e}$ série, $\mathrm{n}^{\circ} 1-2$; et Montaigne et la rhétorique, Actes du colloque de St Andrews, éd. John O’Brien, Malcom Quainton et James Supple, Paris, H. Champion, 1995.

3 Francis Goyet, Le sublime du "lieu commun ". L'invention rhétorique dans l'Antiquité et à la Renaissance, Paris, H. Champion, 1996, p. 389-397.

4 Nous citons les Essais dans l'édition Villey-Saulnier en donnant dans le texte les numéros de livre et de chapitre, ainsi que la pagination de cette édition publiée par les Presses Universitaires de France.

5 Nous reprenons ici la liste de Michel Magnien dans son article «éloquence » du Dictionnaire de Michel de Montaigne, éd. Philippe Desan, Paris, H. Champion, 2004. 
d'école " pour reprendre l'expression de Marc Fumaroli' ${ }^{6}$ Jean Du Bellay, Cléomène, Socrate sont des contre-modèles d'éloquence, emblèmes aussi d'un degré de maîtrise et de naturel incommensurablement plus haut que les personnages auxquels Montaigne les compare. Ils incarnent un niveau de responsabilité politique bien plus élevé, qui seul permet d'allier la maîtrise de la parole à l'attestation d'une justice politique, valeur si chère à Montaigne. À travers ce type d'oppositions, Montaigne se situe dans la lignée de l'anti-rhétoricisme de Platon. Il reproduit l'antagonisme matriciel de Socrate et de Phèdre dans le dialogue qui porte le nom de ce dernier ${ }^{7}$. Mais Socrate n'est pas seulement le détracteur des professionnels de la rhétorique, dont il révèle le manque de hauteur et de profondeur. Il représente également la capacité d'improvisation, qualité suprême de l'orateur ${ }^{8}$, l'éloquence dégagée des règles, par opposition à une éloquence écrite, méthodique et laborieuse - à tous les sens du terme -, qui " pue [...] l'huyle et la lampe» (I, 10, 40).

Le chapitre "De la vanité " amène une autre figure intéressante, un autre repoussoir, Lyncestez, orateur "accablé " sous le poids de sa volonté de bien faire et de son application affectée (III, 9, 962). C'est après son évocation que Montaigne se livre au plus net aveu du caractère profondément artificiel de son apparente négligence : " mon dessein est de representer en parlant une profonde nonchalance et des mouvemens fortuites et impremeditez, comme naissans des occasions presentes " $(963)^{9}$. Montaigne se représente en effet comme stratège de la représentation : c'est le cas ici, mais aussi dans d'autres passages du même chapitre, comme celui qui porte sur les "gaillardes escapades [qui retirent] au nonchalant et au fortuit " (III, 9, 994) ou, dans "De l'institution des enfans ", sur le «bas mal tendu " et la dissimulation des " coutures" (I, 26, 172). Ces pages entrent en tension avec d'autres, notamment l'avis "Au lecteur ", qui revendique une sincérité sinon totale, du moins importante : « je m’y fusse tres-volontiers peint tout entier, et tout nud."

6 Marc Fumaroli, "Rhétorique d'école et rhétorique adulte ; remarques sur la réception en Europe de la réception du traité 'Du Sublime' au XVI" siècle et au XVIII' siècle ", Revue d'Histoire Littéraire de la France, n 1,1986 , p. 33-51.

7 Quintilien traite cette question dans le dyptique que forment les chapitres X, 6, « De la Méditation » et X, 7, "Comment s'acquiert la faculté de parler sur le champ, \& en quoy elle consiste " (De l'institution de l'orateur, trad. N. Gedoyn, Paris, Grégoire Dupuis, 1718). À ce sujet, voir notamment Terence Cave, Cornucopia : figures de l'abondance au XVI siècle, trad. Ginette Morel, Paris, Macula, 1997, p. 308.

8 Quintilien, op. cit., X, 7, 1.

9 À propos de la negligentia diligens, voir entre autres, Hugo Friedrich, Montaigne, trad. Robert Rovini, Paris, Gallimard, 1968, p. 350 ; et Marc Fumaroli, La diplomatie de l'esprit, Paris, Gallimard, 1998, p. 147. 
Je tenterai d'expliciter les présupposés d'une approche rhétorique de Montaigne, puis d'examiner les apports des différents travaux critiques relevant de ce champ de recherche, du point de vue de l'invention, de la disposition et de l'élocution : une des spécificités de l'approche rhétorique consiste à aller des res aux verba, des " choses " aux " mots ». Je reviendrai enfin à la question de la "sincérité".

\section{Présupposés}

Si la critique montaigniste se risque désormais à une approche rhétorique, c'est une certaine conception du texte des Essais qui l'anime et la justifie. Elle conditionne aussi une méthodologie.

Les chapitres des Essais sont tout d'abord conçus comme discours adressés, non pas à la manière de ceux de Cicéron ou de Brutus au Sénat, discours oraux d'un homme politique face à un public physiquement présent. Mais les Essais peuvent être lus comme des discours adressés à l'instar des textes d'Horace, par exemple : telle page est écrite à l'attention de tel ou telle dédicataire, mais l'ensemble de l'œuvre est destiné à un lecteur relativement indéterminé. La question du destinataire est difficile à traiter en ce qui concerne ces deux auteurs ; elle est pourtant fondamentale en rhétorique en général, mais plus encore dans le cas de ces deux auteurs qui tiennent grand compte de leur lecteur. On sait que Montaigne est parfaitement rompu à la lecture d'Horace, qu'il cite souvent, mais aussi de ses nombreux commentaires rhétoriques, dont il possède un bel exemplaire, acquis en $1543^{10}$. Horace incarne un idéal de fiction de conversation avec le lecteur.

Le second présupposé tient à la " contention " des Essais, à l'hypothèse critique selon laquelle les chapitres sont à interpréter comme tendus vers une fin, vers un propos. Si Montaigne évite d'imposer un dogme, d'instruire ouvertement ("Les autres forment l'homme ; je le recite ", III, 2, 804), il " attend de son livre qu'il suscite un éveil, un désir de collaboration $^{11}$ ». Certaines approches valorisent la «promenade » et la gratuité de l'écriture montaignienne ; les chapitres sont alors conçus comme autant de conversations libres plutôt que de discours argumentés ${ }^{12}$. Dès 1908, Gustave Lanson s'est vivement opposé à cette conception très répandue au début du $\mathrm{XX}^{\mathrm{e}}$ siècle :

10 Voir Dorothy Coleman, Montaigne, quelques Anciens et l'écriture des Essais, Paris, H. Champion, 1995, p. 19 sq.

11 Christian Mouchel, «Les rhétoriques post-tridentines (1570-1600) : la fabrique d'une société chrétienne ", in Histoire de la rhétorique dans l'Europe moderne : 1450-1950, éd. Marc Fumaroli, Paris, Presses Universitaires de France, 1999, p. 483.

12 Voir entre autres Hugo Friedrich, Montaigne, op. cit., p. 348 et Nicola Panichi, La virtù eloquente : la "civil conversazione " nel Rinascimento, Urbino, Montefeltro, 1994. 
Montaigne, à le prendre dans l'idée banale qu'on s'en fait couramment, est un nonchalant qui couche toutes ses fantaisies par écrit, comme elles lui viennent. [...] Ce n'est pas le vrai Montaigne. Sans parler de l'effort sérieux, puissant de pensées que la richesse et la profondeur des Essais révèlent - on ne va pas si loin en s'amusant, sans le vouloir, - le style n'est pas une causerie négligemment improvisée. Les négligences sont voulues, du moins acceptées avec réflexion. ${ }^{13}$

Il n'est pas certain que ces deux conceptions s'excluent réciproquement. L'une met en lumière la gratuité de l'œuvre de Montaigne, l'autre, sa dimension argumentative. Peut-être ce problème critique se pose-t-il d'ailleurs dans les mêmes termes à propos d'Horace, dont certains passages semblent chercher à convaincre le lecteur d'un credo moral alors que d'autres donnent une impression de conversation libre (sermo), vagabonde ou "à vau-l'eau " pour parler en termes montaigniens. À la lecture de Montaigne comme à celle d'Horace, tout est question de pondération ; tout repose sur l'importance accordée à chacun de ces deux éclairages qui peuvent être portés sur les Essais.

On pourrait également les rapprocher de l'œuvre virgilienne. Marc Fumaroli les qualifie de "Géorgiques de la parole : tout au rebours de la surface lisse et stérile de l'élocution cicéronienne ${ }^{14}$ ». Rappelons tout de même que les Géorgiques sont imprégnées de rhétorique. Montaigne possède d'ailleurs une édition de la trilogie virgilienne encadrée des commentaires rhétoriques très fins de Nicolaus Erythraeus ${ }^{15}$. Les œuvres virgilienne et montaignienne, parmi d'autres, conduisent à dépasser de nombreuses dichotomies, notamment le pseudo-clivage entre rhétorique et poétique.

Le texte montaignien s'inscrit dans une relation d'allocution et, à ce titre, peut être abordé de la même manière que des textes transposant à l'écrit des discours oraux, historiques, comme ceux de Cicéron, ou fictionnels, comme les œuvres de Térence ${ }^{16}$ et de Virgile, que Montaigne ne lisait pas nus, mais habillés de leurs commentaires rhétoriques. Étant bien plus descriptive que prescriptive, l'approche rhétorique moderne ressemble par bien des côtés à la démarche critique de commentaires dont Montaigne était parfaitement familier.

13 Gustave Lanson, L'art de la prose [1908], Paris, Fayard, 1920, p. 45, cité par Alexandre Tarrête, Les Essais de Montaigne, Paris, Gallimard, 2007, p. 228.

14 Marc Fumaroli, La diplomatie de l'esprit, op. cit., p. 137.

15 P. Virgilii Maronis Bucolica, Georgica et Aeneis, nunc demum Nicolai Erythraei [...], Venetiis, apud F. Rampazetum, expensis M. Sessae, c. 1539.

16 Montaigne disposait de deux éditions des commentaires de Donat à propos de Térence, l'une d'Érasme et l'autre de Robert Estienne : Habes hic amice lector P. Terentii Comoedias, una cum scholiis ex Donati, Asperi, [et] Cornvti commentariis decerptis, studio et opera Des. Erasmi Roterodami, Basileae, in officina Frobeniana, 1538 ; Terentii Comoediae sex, tum ex Donati commentariis, studio et opera Des. Erasmi Roterodami, Parisiis, ex officina R. Stephani, 15411543. 


\section{Invention}

L'invention est le premier terrain de jeu de l'esprit ingénieux ; on comprend donc l'importance que lui accorde Montaigne. Perçus comme des « Discours moraux, politiques et militaires ", selon le titre de leur première traduction italienne, les Essais de 1580 laissent transparaître un dessein d'utilité. L'approche historique et sociologique leur assigne souvent une causa dicendi précise : la modération des passions humaines dans le cadre des guerres de religion que le roi ne parvient pas à calmer. La rhétorique se nourrit des travaux historiques. Mais l'intention utile, donc délibérative, n'en est certainement qu'un des deux versants, pour reprendre l'adage horatien, Omne tulit punctum qui miscuit utile dulci $i^{17}$. Nous l'examinerons sous ses deux pans, l'utile et l'agréable.

Le premier aspect de l'invention montaignienne tient donc à son utilité politique et morale, qui se traduit par la contention évoquée plus haut. Les sujets qu'aborde Montaigne ne se distinguent pas par leur nouveauté fracassante ; ayant souvent rapport à la vertu, nombre d'entre eux sont des sentiers battus de la morale antique, comme les thèmes de la colère ou de la cruauté, ce qui explique en partie la prolifération des citations latines dans l'œuvre - la rhétorique dialogue aussi avec la philosophie morale et politique. La manière dont Montaigne les traite est originale, puisqu'il en forme un pot-pourri, un pastissage, et qu'il cultive le décalage entre leur sens originel de la citation et celui qu'elles ont dans le chapitre des Essais qui les sertit. Les lieux communs sont l'objet d'un " processus de variation ou de transformation » : « le rejet des évidences, des lieux communs rebattus mène au comble de la singularité ». L'invention montaignienne rebondit sur ces citations ou sur ces allusions, par les pratiques de la glose, du commentaire et du paradoxe ${ }^{18}$.

Marc Fumaroli a montré que l'œuvre est également orientée vers l'auteur lui-même, cherchant à favoriser "une sorte de 'révélation' naturelle ». Elle conduit au «façonnement de l'auteur par l'invention de son propre discours, comme si celui-ci avait pour première fin non de convaincre autrui, mais de faire jaillir une vérité cachée au fond de l'âme ${ }^{19}$ ". Cette démarche n'est pas sans rappeler la figure de Socrate, sorte de crête surmontant les deux versants que nous avons distingués.

17 Horace, De arte poetica, v. 343 : «il enlève tous les suffrages celui qui mêle l'agréable à l'utile ».

18 Voir Terence Cave, op. cit., p. 281-289 ; Ann Moss, Les recueils des lieux communs: méthode pour apprendre à penser à la Renaissance, trad. éd. P. Eichel-Lojkine, Genève, Droz, 2002, p. 331 ; Francis Goyet, "Montaigne et les recueils de lieux dits communs", in Normativités $d u$ sens commun, éd. Claude Chappe-Gautier et Sandra Laugier, Paris, Presses Universitaires de France, 2009, p. 51-93 ; et André Tournon, Montaigne. La glose et l'essai, Lyon, Presses universitaires de Lyon, 1983.

19 Dans ce passage (L'Âge de l'éloquence, op. cit., p. 644-645), Marc Fumaroli rapproche l'écriture de Montaigne de celle de l'abbé de Saint-Cyran, auteur postérieur. 
L'écriture montaignienne des Essais repose également sur le doux, l'agréable, le plaisir partagée ${ }^{20}$, donc sur le relâchement de la tension argumentative. François Rigolot, Olivier Guerrier et Nathalie Dauvois ont montré le rôle que jouent les fictions narratives comme celle d'Ovide et d'Ésope, de Virgile, dans l'invention montaignienne : le commentaire, l'induction, l'élargissement et la similitude en sont quelques ferments ${ }^{21}$. Les Essais traitent aussi de nombreux sujets s'inscrivant dans le champ d'intérêts du gentilhomme de l'époque : les questions d'entregent, d'ambassade, en particulier dans le premier livre, le courage, les grands hommes de son temps, les faits et gestes des grands hommes du passé, le voyage. Francis Goyet a souligné le rôle que jouent les exemples historiques dans la captatio benevolentiae, et synthétisé l'éthique du gentilhomme que présentent notamment les Essais; celle-ci repose sur une certaine représentation de l'audace, du processus décisionnel, de l'« humaine prudence», de l'habitus, du jugement et de l'orgueil ${ }^{22}$.

La représentation de l'auteur est un élément important de l'éthos, autrement dit de la conciliation. Le portrait de l'auteur, portrait qui miscuit utile dulci, pourrions-nous dire, joue un rôle crucial dans l'œuvre. Les Essais entreprennent une justification, une auto-apologie, qui fonde l'autorité du discours tout autant qu'elle incarne. Le discours sur soi nourrit la réflexion morale. La crédibilité de l'auteur en apparaît comme l'enjeu principal. Montaigne ne se représente pas seulement comme "de bonne foy" (selon le topos de la bona fides, qui marque le seuil de l'œuvre), mais aussi comme sympathique, supérieurement sage et supérieurement modeste. Il s'ingénie à séduire son lecteur par son humilité. Les approches rhétoriques tendent à valoriser l'ambiguïté dont sa modestie est empreinte ${ }^{23}$; elles rappellent que cette humilité relève du lieu commun, en l'occurrence de l'excusatio propter infirmitatem. Mais l'agrément du texte des Essais se manifeste également dans leur progression logique et thématique.

20 Voir Yvonne Bellenger, "Montaigne et la douceur ", La douceur en littérature : de l'Antiquité au XVIIe siècle, éd. Hélène Baby et Josiane Rieu, Paris, Classiques Garnier, 2012, p. 35-48 ; et Michel Magnien, "Légèreté, plaisir et désinvolture : Montaigne à l'école de Martial ? ", Montaigne Studies, vol. XVII, 2005, p. 97-118.

21 François Rigolot, Les Métamorphoses de Montaigne, Paris, Presses Universitaires de France, 1988 ; Nathalie Dauvois, Prose et poésie dans les Essais de Montaigne, Paris, H. Champion, 1997 ; Olivier Guerrier, Quand "les poètes feignent " : "fantasie " et fiction dans les Essais de Montaigne, Paris, H. Champion, 2002, en particulier p. 57-74.

22 Francis Goyet, Les Audaces de la Prudence. Littérature et politique aux XVIe et XVII siècles, Paris, Classiques Garnier, 2009, et " La séduction par les exemples (historiques) : le démarrage des Essais ", Journée d'étude L'Exemple en rhétorique, 12 mai 2011, éd. E. Danblon, Bruxelles, Presses de l'Université Libre de Bruxelles, 2014.

23 Lawrence D. Kritzman, Destruction-découverte : le fonctionnement de la rhétorique dans les Essais de Montaigne, Lexington, French Forum, 1980, p. 110-115 ; et Erich Auerbach, Mimésis. La représentation de la réalité dans la littérature occidentale, trad. C. Heim, Paris, Gallimard, 1968, p. 297 sq. Voir aussi Valérie Dionne, Montaigne, écrivain de la conciliation, Paris, Classiques Garnier, 2014. 


\section{Disposition}

L'auteur manifeste la pertinence de son jugement par la disposition qu'il adopte, puisqu'on ne peut juger de la valeur d'un argument qu'en fonction de sa place dans le discours. "(Car quand à l'ordre, je sens bien que je le trouble, mais je n'en observe non plus à renger ces exemples qu'au reste de toute ma besongne) [...]" (II, 12, 465) : cette parenthèse digressive met en abyme la négligence, la diligente négligence, dont il est question. Montaigne se garde bien d'adopter une disposition trop canonique, ou trop cicéronienne et clame incessamment son refus d'une collocation calculée ${ }^{24}$. Plus encore que sur le plan de l'invention, c'est sur celui de la disposition qu'il laisse le plus libre cours aux " chimeres et monstres fantasques [de son esprit], sans ordre, sans propos " (II, 8, 33) et à la " fortune ». Ces protestations de négligence n'ont pas dissuadé la critique de chercher un dessein et un ordre dans la progression de tel ou tel chapitre.

Les travaux de Peter Mack et les miens présentent des démarches relativement comparables puisque nous commençons par repérer des paragraphes dans le flux du chapitre étudié ${ }^{25}$, en partant de la première édition du chapitre, pour examiner ensuite les ajouts longs. L'analyse rhétorique des Essais fait donc souvent intervenir des considérations philologiques. À propos du chapitre "Des menteurs ", Peter Mack rappelle par exemple que la combinaison de séquences narratives et de maximes renvoie à une pratique de collège, parce que les élèves étaient entraînés à développer conjointement des maximes et des illustrations historiques. Ses analyses replacent le texte dans la culture rhétorique de son temps, qui est celle de l'auteur, mais aussi celle du lecteur que Montaigne se représente probablement.

La dialectique et la rhétorique distinguent deux types d'ordre. Ramus oppose, dans la lignée d'Aristote, "méthode de nature " et " méthode de prudence ${ }^{26} »$. Le fait qu'un discours relève de l'un ou de l'autre est absolument déterminant, en particulier pour Montaigne. La méthode de nature, autrement dénommée méthode d'art, vise à conduire le plus clairement possible le lecteur dans le discours, par une annonce du propos et du plan, divers rappels de la structure du discours, des transitions, une

24 Voir, entre autres, André Tournon, Montaigne. La glose et l'essai, op. cit., p. 124-145; et Francis Goyet, "Les Essais entre marqueterie 'mal jointe' et nid 'bien joint"', Montaigne Studies, vol. XXVI, 2014, p. 37-54.

25 Peter Mack, Reading and rhetoric in Montaigne and Shakespeare, Londres, Bloomsbury, 2010, chapitre 3 ; Paul J. Smith, Dispositio : Problematic Ordering in French Renaissance Literature, Leiden, Brill, 2007, chap. 9 et chap. 10 ; et Déborah Knop, "La Cryptique chez Montaigne ", Thèse de doctorat, dir. F. Goyet, soutenue à l'Université Stendhal Grenoble Alpes le 8 décembre 2012.

26 Pierre de La Ramée, Dialectique, Paris, André Wéchel, 1555, deuxième livre. 
progression du général au particulier. Dans ma thèse à paraître, j’ai cherché à montrer que Montaigne répugne souvent à ce type d'écriture, sans pour autant que la progression du discours soit aléatoire. Les Essais se situent souvent dans la méthode de prudence, qui présuppose un lecteur réticent. Quelques manipulations (suppression, ajout, inversion) à partir de l'ordre " naturel », celui qui favorise la compréhension et la mémorisation, suffisent pour dérouter le lecteur. Il perd alors le fil. Même dans les chapitres dont la progression semble décousue, il existe une ligne conductrice, mais celle-ci est cachée : "C'est l'indiligent lecteur qui pert mon subject, non pas moy ». Ici encore, tout est question de pondération, et peut-être aussi de diligence. Les écarts par rapport à l'ordre naturel qui aurait favorisé la clarté peuvent concerner le début du chapitre, autrement dit son titre et ses premiers mots, potentiellement insinuants, le corps de l'argumentation - on parle alors de digression -, ou sa $f^{27}{ }^{27}$. Si la science rhétorique peut mettre en lumière la dissimulation à l'œuvre dans les Essais, celle-ci n'est pas à entendre comme une forme de mensonge ni d'hypocrisie. Elle n'a rien de fallacieux. L'écrivain ne cache que pour dévoiler, pour que son lecteur prenne conscience d'une idée importante, le plus souvent d'ordre moral ou politique. Il cherche à lui ouvrir les oreilles et le cœur avant de révéler son propos, plutôt que de se heurter à sa résistance. Ainsi entendue, la dissimulation s'apparente le plus souvent à un procédé de retardement (commoratio). De nombreux éléments stylistiques entrent en compte.

\section{Élocution}

C'est la problématique de l'élocution qui a, sans conteste, attiré le plus d'études. Pourtant, les grands traités antiques tendent à minorer son importance au regard de l'invention et de la disposition. "Je veux donc que l'on pense aux mots [élocution], mais que l'on soit beaucoup plus occupé des choses [invention] ${ }^{28}$ ». L'élocution, qui découle naturellement des deux premières parties de la rhétorique, ne doit donc pas focaliser toute notre attention : "L'éloquence demande un esprit plus masle, \& lorsqu'elle est saine \&

27 Marcel Tetel, «Les fins d'essais : la mise en question ou début du convaincre ", in Rhétorique de Montaigne, op. cit., p. 191-202 ; Jean-Paul Sermain, "Insinuatio, circonstantia, visio et actio : l'itinéraire rhétorique du chapitre III, 4, 'De la Diversion' ", in Rhétorique de Montaigne, p. 123-139 ; André Tournon, Route par ailleurs, Le "nouveau langage " des Essais, Paris, H. Champion, 2006, p. 62-78 et 79-114 ; Gérard Milhe-Poutingon, Poétique du digressif : la digression dans la littérature de la Renaissance, Paris, Classiques Garnier, 2012 ; et Déborah Knop, "La conciliatio, entre exorde et digression : Montaigne, Essais, II, 25, 'De ne contrefaire le malade' ", in L'Art de la conciliation, Genève, Droz, "Les Cahiers du Gadges ", $\mathrm{n}^{\circ} 11,2013$, p. 76-89.

28 Quintilien, op. cit., VIII, 20. 
vigoureuse, il ne luy faut point tant de frisure ni de façon ${ }^{29}$ ", ajoute Quintilien dans un passage aux accents montaigniens. Toujours est-il que les Essais réfléchissent souvent (à) leur forme, leur langue et leur style.

Les idées des Quintilien, notamment sa valorisation de l'enargeia et du pectus, innervent l'esthétique des Essais. La position de Montaigne vis-à-vis de Cicéron est plus complexe. Dans son apparente allergie à l'éloquence cicéronienne, Montaigne s'inscrit dans un débat de son temps, et dans la continuité du Ciceronianus d'Érasme (1528), comme l'explique Michel Magnien ${ }^{30}$. L'éloquence montaignienne, celle du sermo humilis, rhétorique " de la bassesse ", ne renonce pas pour autant au sublime ; propre à "l'otium ", elle n'abdique pas non plus devant une possible action sur la sphère des negotia, dont elle s'éloigne ou prétend s'éloigner ${ }^{31}$.

Pour Montaigne, Cicéron incarne les préceptes de l'éloquence appliqués de manière mécanique et par trop prévisible, notamment à cause de "ses longueries d'apprêts » (II, 10, 413) et de l'usage pédagogique qui en est fait au $\mathrm{XVI}^{\mathrm{e}}$ siècle $^{32}$. Les Essais assimilent volontiers la rhétorique scolaire et pédante à la figure du "pere d'éloquence " (II, 31) - et réciproquement (I, 40 ; I, 26 ; II, 10 ; II, 12 ; II, 17 ; II, 31). Cette réduction ne va pas sans contradictions. Dans "Des livres ", Montaigne écrit à propos de Cicéron, en citant Brutus, "que c'estoit une eloquence cassée et esrenée, fractam et elumbem " et, quelques lignes plus bas, qu' " elle est du tout hors de comparaison ; je croy que jamais homme ne l'egalera " (II, 10, 415). C'est vraisemblablement un symbole de "vanité » et de "paroles creuses ${ }^{33}$ " que Montaigne fustige en Cicéron, et non pas l'éloquence cicéronienne elle-même. Il faut relire Cicéron en regard de Montaigne pour mesurer l'influence de l'un sur l'autre : dans un article récent, Eric MacPhail a montré que le "stile comique et privé " des Essais était placé sous l'égide des Lettres familières de Cicéron ${ }^{34}$.

29 Ibid., VIII, 22. Voir aussi Gisèle Mathieu-Castellani, «L’intertexte rhétorique:Tacite, Quintilien et la poétique des Essais ", in Montaigne et la rhétorique, op. cit., p. 17-26 ; Gérard Defaux, "Inter otia animorum et spes inanes: Quintilien, La Boétie, Montaigne ", Bibliothèque d'Humanisme et Renaissance, vol. LIX, n 3, 1997, p. 513-535 ; Gérard Defaux, article "Quintilien ", et M. Magnien, article " éloquence " du Dictionnaire de Michel de Montaigne, op. cit.

30 Michel Magnien, "D’une mort, l'autre (1536-1572) : le rhétorique reconsidérée ", in Histoire de la rhétorique dans l'Europe moderne, op. cit., en particulier p. 394 ; et «Un écho de la querelle cicéronienne à la fin du XVI e siècle : éloquence et imitation dans les Essais ", in Rhétorique de Montaigne, op. cit., p. 85-99; Mary B. McKinley, «La présence du Ciceronianus dans 'De la vanité" ", in Montaigne et la rhétorique, op. cit., p. 51-65.

31 Voir Christian Mouchel, art. cit., p. 482 sq. ; Francis Goyet Le sublime du "lieu commun", op. cit., p. 691-704; Michel Magnien, "Montaigne et le sublime dans les Essais», in Montaigne et la rhétorique, p. 27-48; et Floyd Gray, Le style de Montaigne, Paris, A.- G. Nizet, 1992, p. 74.

32 Voir Élie Vinet et André de Gouvea, Schola Aquitanica, Bordeaux, S. Millanges, 1583.

33 Michel Magnien, article "Cicéron" du Dictionnaire de Michel de Montaigne, op. cit.

34 Eric MacPhail, "Considerations on Cicero and Montaigne ", Montaigne Studies, vol. XXVII, 2015, p. 11-21. 
Le rejet de l'esthétique cicéronienne et l'éloge, voire l'adoption, d'une esthétique plus sénéquienne correspondent à des codes sociaux aristocratiques. De ce fait, ils relèvent de la convenance ${ }^{35}$; Montaigne met en avant d'autres modèles oratoires pour se positionner dans le même débat : Brutus et Sénèque, deux modèles de simplicité, de densité, de rapidité et d'énergie. Brutus apparaît sous la plume de Montaigne comme homme d'action plus que de lettres (voir II, 10, 415 ; II, 31, 716 ; II, 34, 736 ; III, 9, 996 ; et III, 13, 1108), parangon de la profondeur, de l'audace, de la rhétorique du cœur ou du pectus (II, 31, 716), alors même qu'aucun de ses textes ni de ses discours de nous est parvenu ${ }^{36}$. Quant à Sénèque, au XVI ${ }^{\mathrm{e}}$ siècle, il est érigé en modèle du " questionnement apparemment décousu ", " des répliques acérées ${ }^{37}$ " et, sous la plume de Montaigne, en incarnation de la virilité stylistique : Non est ornamentum virile concinnitas (I, 40, 251, "Ce n'est pas un élément viril que l'élégance ", Sénèque, Lettres à Lucilius, CXV, 2). La lettre 115 des Épitres semble effectivement incontournable pour comprendre l'esthétique montaignienne : "Chaque fois que tu verras un style laborieusement raffiné, sache que l'âme de l'auteur est tout occupée ellemême pareillement de mesquineries. Le langage de l'âme vraiment grande est plus rassis, plus calme ; tous ses propos témoignent de plus d'assurance que d'apprêts ${ }^{38}$ \%. Sénèque poursuit par un portrait physique, littéraire et moral des " élégants ». Le style ne vaut pas en tant que tel ; si Sénèque et Montaigne lui accordent une telle attention et s'y fient à ce point, c'est en tant que révélateur d'une réalité morale.

Montaigne utilise de telles figures auctoriales pour mettre en exergue ses propres valeurs d'authenticité, son inéluctable volonté de cohérence entre les écrits et les faits, entre identité privée et présence publique. Elles lui permettent de ciseler un éthos d'auteur fiable, honnête et viril. Ces protestations valent autant pour leur caractérisation des Essais qu'en tant que critiques implicites visant certains de ses contemporains.

Le débat sur le style périodique, lié ou coupé, est à comprendre dans son lien avec la querelle du cicéronianisme, puisque Montaigne attache usuellement à Cicéron les liaisons et les nombres propres aux canons antiques. Jean Lecointe a retracé l'opposition entre les tenants de celui-ci, imité du latin, et les partisans

35 Déborah Knop, "Montaigne and Rhetoric ", in Oxford Handbook of Montaigne, éd. Philippe Desan, New York, Oxford University Press, 2016.

36 Montaigne aborde d'ailleurs ce paradoxe (II, 10, 415).

37 Voir Ann Moss, op. cit., p. 368. Celle-ci s’appuie sur Edmond Richer, défenseur de Cicéron dans ladite querelle, qui formule des objections à l'engouement pour Sénèque (Obstetrix animorum, Paris, A. Drovart, 1600). À propos de l'opposition entre les styles de Tacite et de Sénèque dans les Essais, voir Paul J. Smith, op. cit., p. 198-199. À propos de l'influence virile de Sénèque sur le style montaignien, voir Maria Protopapas-Marnelli, Montaigne, la vigueur du discours : sur une influence de rhétorique stoücienne dans les Essais, Laval, Presses de l'Université de Laval, 2009, 28 sq. et 100.

38 Sénèque, Entretiens, Lettres à Lucilius, Paris, Robert Laffont, 1993, p. 1045. 
du second, qui semble plus naturel ; entre le principe de la liaison et celui de la coupure. Il montre que la forme périodique structure tout de même le " paragraphe " montaignien, ou du moins " certains enchaînements » argumentatifs ; le naturel du style coupé cherche à éviter que des structures syntaxiques et stylistiques trop visibles ne se forment, mais la clausule cicéronienne est encore très présente; elle introduit un effet de ponctuation finale efficace ${ }^{39}$.

Thérèse Le Flanchec a prolongé l'étude de Jean Lecointe pour étudier les caractéristiques syntaxiques et stylistiques de cette écriture : effets d'attente de la clausule qui referme la période, suppression des connecteurs logiques et des corrélatifs (parataxe), recours limité à la subordination, phrase simple, ellipse verbale, énumération, phénomènes d'extraction et de dislocation, hyperbate ${ }^{40}$. Violaine Giacomotto a approfondi l'analyse de l'"efficacité de la langue ", notamment les ressorts syntaxiques de la concision. L'emphase sous-tend de nombreux passages des Essais, sur le plan grammatical, mais aussi stylistique ${ }^{41}$.

Pour reprendre une problématique abordée en début d'article, rhétorique et poétique ne sont pas incompatibles. Montaigne recourt à de nombreuses figures qui poétisent le discours et qui sollicitent l'imagination en renforçant l'argumentation : répétition, métaphore, allégorie, similitude et comparaison, paronomase, signification, prétérition, prosopopée ${ }^{42}$, entre autres, sont récur-

39 Jean Lecointe, "L'organisation périodique du 'style coupé' dans le livre III des Essais ", in Styles, genres, auteurs: Montaigne, Bossuet, Lesage, Baudelaire, Giraudoux, éd. A.-M. Garagnon, Paris, Presses de l'Université Paris-Sorbonne, 2001, p. 9-24 ; et Lidéal et la différence : la perception de la personnalité littéraire à la Renaissance, Genève, Droz, 1993, p. 606 sq.

40 Thérèse Le Flanchec, op. cit., p. 263 sq. Voir aussi Françoise Charpentier, «L'hyperbate : une maitresse forme du troisième allongeail ", in Montaigne et l'accomplissement des Essais, Bulletin de la Société des Amis de Montaigne, juillet-déc. 1988-janv.-juin 1989, p. 129-147.

41 Emmanuel Naya et Violaine Giacomotto-Charra, Essais de Montaigne. Livre I, Paris, Atlande, 2010, p. 150 sq. et p. 171 sq. Violaine Giacomotto-Charra souligne entre autres l'importance que jouent la détermination zéro des noms communs et l'attribut de l'objet dans la concision du style montaignien. Concernant toutes les questions évoquées dans cette partie, voir aussi le riche volume consacré à La langue de Rabelais, la langue de Montaigne, op. cit. : les articles de Bernard Combettes et de Gérard Milhe Poutingon, portent sur la question de la mise en relief grammaticale. Concernant l'emphase stylistique, voir Margaret McGowan, «L'hyperbole dans les Essais», in Montaigne et la rhétorique, op. cit., p. 99-114; et Déborah Knop, «L'amplification chez Montaigne : une question d'optique, et de jugement (Essais, III, 6, "Des coches") ", in L'optique des moralistes, éd. Bernard Roukhomovsky, Paris, H. Champion, 2005, p. 393-404.

42 Divers articles des volumes Rhétorique de Montaigne et Montaigne et la rhétorique traitent de ces questions. Voir aussi Emmanuel Naya et Violaine Giacomotto-Charra, op. cit., p. 164-169, à propos de la répétition ; Michel Magnien, "'Tel [...] faict des Essais qui ne sauroit faire des effaicts' : la paronomase dans les Essais ", Montaigne Studies, vol. XXVIII, 2015, p. 113-126; Gisèle Mathieu-Castellani, « Dire, Signifier : la figure de la Significatio dans les Essais», Montaigne Studies, vol. III, 1991, p. 68-81 ; Blandine Perona, Prosopopée et persona à la Renaissance, Paris, Classiques Garnier, 2013, p. 205-295 ; André Tournon, "Les prétéritions marquées ou le sens de l'inachèvement ", Bulletin de la Société des Amis de Montaigne, VII" série, nº 13-16, juil.-déc. 1988-janv.-déc. 1989, p. 231-238. 
rentes dans les Essais et déterminantes en termes de style. L'étude de la ponctuation et des majuscules dites " de scansion " permet d'observer le rythme du texte et certains effets d'insistance ; elle est à relier à l'expression des affects et des émotions ${ }^{43}$, question centrale des études montaignistes. L'analyse rhétorique propose de rattacher l'esthétique générale des Essais à des points de langue très particuliers.

\section{La question de la « sincérité »}

Les approches rhétoriques sont multiples, et on a souvent pu leur attribuer une conception schématique du texte montaignien qui ne vaut certainement pas pour toutes. La critique a souvent projeté en l'approche rhétorique une interprétation qui soupçonnerait une certaine duplicité montaignienne. Or il ne nous semble pas que le prisme de la rhétorique conduise nécessairement à percevoir les Essais comme un texte qui ne serait pas sincère. Il n'est d'ailleurs pas certain que la rhétorique conduise à raisonner en ces termes. Le problème de la transparence ou de la sincérité pourrait se poser ainsi au barreau, puisque le genre judiciaire, orienté vers des faits passés, s'évalue selon les critères du vrai et du faux. Mais les Essais s'apparentent plutôt aux genres démonstratif et délibératif ; peut-être nous invitent-ils alors à raisonner autrement.

La dimension démonstrative des Essais introduit une réflexion atemporelle et plutôt théorique, qui repose sur les notions de bien et de mal ${ }^{44}$. On peut également les envisager à la lumière de la délibération, comme tournés vers l'avenir et régis par les critères de l'utile et du nuisible. Dans les deux cas, le prisme rhétorique révèle une intention des Essais, la tentative de modeler ou de faire émerger une conscience politique et morale. Le discours montaignien est nécessairement sincère, dès lors; non pas au sens d'une éventuelle transparence autobiographique - question que l'art rhétorique ne se pose guère, et qui reviendrait peut-être à d'autres disciplines -, mais dans un sens psychagogique : le texte esquisse une progression morale vers une vertu sincèrement envisagée comme horizon. La "bonne foy " de l'auteur est de nature atemporelle ou, si nous nous permettons un latinisme, crastique.

43 Nous renvoyons aux travaux d'A. Tournon sur ces questions.

44 À propos du genre démonstratif, voir Michel Simonin, L'encre et la lumière, Genève, Droz, 2004, III, 1, « Poétique(s) du politique, Montaigne et Ronsard prosopographes de François de Guise », p. 407 sq. ; David Quint, "Montaigne et Henri IV », in L'éloge du prince : de l'Antiquité au temps des Lumières, éd. Isabelle Cogitore et Francis Goyet, ELLUG, 2003, p. 250-260 ; et Claudie Martin-Ulrich, "Malaise de l'éloge : considération sur Montaigne ", in "Eveils ", Études en l'honneur de Jean-Yves Pouilloux, éd. V. Fasseur, O. Guerrier, L. Jenny et A. Tournon, Paris, Classiques Garnier, 2010, p. 101-115. 
Sans les priver de la littérarité qui leur est propre, l'approche rhétorique permet d'attirer l'attention sur divers aspects des Essais et d'approfondir leur analyse, à commencer par la tension argumentative de tel ou tel chapitre ou du moins de tels et tels passages, et en définitive de l'ensemble de l'œuvre. La rhétorique offre également des éléments d'analyse pour commenter la progression du chapitre, postulant que, le plus souvent, elle n'est pas complètement aléatoire. Elle permet aussi de montrer comment les dimensions poétique et rhétorique s'entrelacent dans les Essais, en référence entre autres à l'œuvre horatienne. Le style de Montaigne peut s'étudier en regard des grands modèles de l'éloquence (Cicéron, versus Sénèque, César notamment), et être resitué dans un débat de son temps. L'angle rhétorique complète enfin une approche autobiographique car elle porte une autre lumière sur le discours sur soi. La rhétorique conduit peut-être à déplacer le problème de la sincérité et de lire autrement ce discours.

Certes, Montaigne souligne souvent la part de la fortune dans sa composition littéraire, et son caractère "imprémédité ". Ce sont deux aspects essentiels de son écriture. Ces conceptions très anciennes ont une légitimité évidente - elles la puisent entre autres dans le texte lui-même. L'approche rhétorique, qui est, en tant que prisme, nécessairement partiale et nécessairement partielle, éclaire un autre aspect du texte. Elle s'appuie notamment sur les éléments de la culture et de la formation littéraires de l'époque, sur des lectures que Montaigne a faites, au collège notamment, et qu'il a pour beaucoup prolongées par la suite.

Déborah Knop Équipe RARE, Université Grenoble Alpes

\section{Résumé}

Si la critique montaigniste se risque, depuis quelques décennies, à une approche rhétorique, c'est une certaine conception du texte des Essais qui l'anime : les chapitres des Essais sont conçus comme discours adressés et tendus vers une fin argumentative. Les approches rhétoriques conditionnent aussi une méthodologie, de l'étude de l'invention à celle de l'élocution. Soulignons toutefois leur grande diversité, notamment au regard de certaines problématiques essentielles des Essais, comme la question de la sincérité.

\section{Mots-clés}

Représentation de soi, politique, morale, invention, disposition, élocution, sincérité.

\footnotetext{
Abstract

Specialists of Montaigne have traditionally used the rhetorical approach to the Essais by considering that chapters were designed as speeches addressed to the reader and organized around an argumentative purpose. Indeed, rhetorical approaches presuppose a methodology, starting from the study of concepts such as inventio and elocutio. However, in contrast to these canonical readings, I also emphasize their large diversity, in particular in relation to some key problems specific to the Essais, for example the question of sincerity.
} 
Keywords

Self-representation, politics, moral, inventio, dispositio, elocutio, sincerity. 